\title{
Experimental investigation of an optimised pitch control for a vertical-axis turbine
}

ISSN 1752-1416

Received on 26th March 2019 Revised 2nd August 2019 Accepted on 23rd October 2019 E-First on 21st November 2019 doi: 10.1049/iet-rpg.2019.0309 www.ietdl.org

\author{
Shokoofeh Abbaszadeh ${ }^{1}$, Stefan Hoerner ${ }^{2,3}$, Thierry Maître ${ }^{3}$, Roberto Leidhold ${ }^{1}$ \\ ${ }^{1}$ Department of Electrical and Information Engineering, University Otto-von-Guericke, Magdeburg, Germany \\ ${ }^{2}$ Department of Process and Systems Engineering, Institute of Fluid Dynamics and Thermodynamics, University Otto-von-Guericke, Magdeburg, \\ Germany \\ ${ }^{3}$ Department of Engineering, University Grenoble Alpes, CNRS, Grenoble-INP, LEGI, Grenoble, France \\ 凶-mail: abbaszadeh@ovgu.de
}

\begin{abstract}
This study reports on an experimental method to obtain the motion law of a pitch system required for maximising the power output of a vertical-axis water turbine (VAWT). In order to allow for the close monitoring of blade forces, the turbine is modelled with a single oscillating hydrofoil in a water channel. The pitch motion is controlled numerically, and the pitch angle and forces are measured as a function of time. The objective of the optimisation is to maximise the time integral of the thrust coefficient. The optimisation is based on a hardware-in-the-loop method and uses a multi-scale, full-factorial approach. The optimal motion law is found through successive improvements with an adaptive parameter step refinement, while the integral of the driving force coefficient is used as a target function. An optimal pitch trajectory with a significant improvement in the thrust coefficient was obtained for a single operating point. The benefit of this methodology is the possibility to study a complete range of turbine designs through the variation of two dimensionless parameters (tip-speed ratio $\lambda$ and reduced frequency $k$ ), within very short runtime, minimising time and costs investigation, compared to more common numerical methods, or experiments on a complete VAWT.
\end{abstract}

\section{Introduction}

Hydropower is the largest source of renewable energy worldwide and often deemed as an ecological and sustainable technology. Nevertheless, the sustainability of hydropower energy strongly depends on its design and the implementation of the facility. Most of the conventional turbines need a high water head and a dam to operate, which have negative impacts on the environment, flora and fauna, but also social impacts like land loss and relocation of the local population. Hydrokinetic turbines, however, are sustainable energy sources. They directly convert the fluid kinetic energy without the need for crosswise structures such as dams or weirs. Moreover, they are cost effective with simple construction and lower the impact on the environment. The obvious drawback

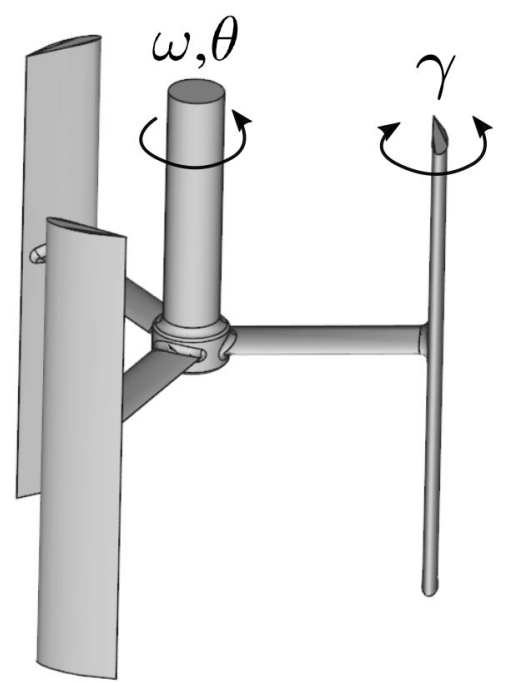

Fig. $13 D$ model of a hydrokinetic, three-bladed, vertical-axis Darrieus water turbine (straight bladed, solidity $\sigma=1$ and turbine aspect ratio $A=1)$ with angular velocity $(\omega)$, azimuth angle $(\theta)$ and pitch angle $(\gamma)$ [3] of these turbines is their low power density and low efficiency compared to conventional solutions. Nevertheless, they can become an interesting solution, especially in tidal and marine applications, installed in water turbine farms.

Among hydrokinetic turbines, vertical-axis water turbines (VAWTs), also known as cross-flow turbines (see Fig. 1), feature some remarkable advantages compared to horizontal-axis systems. They operate independently from the flow direction, which make them excellent candidates for exploiting tidal currents or unsteady flows. Furthermore, they are less sensible to wake effects and the area-based power density for wind farms is significantly higher compared to other kinetic turbines [1], a gain of factor 10 compared to horizontal-axis competitors was reported by Dabiri [2]. However, VAWTs suffer from vibrations and fatigue cracks due to their complex fluid mechanics, which feature alternating loads and dynamic stall. As a consequence of the vertical rotation axis, the free-stream velocity and the blade velocity led to a relative reference frame (speed triangle) with alternating both angle of incidence $(\alpha)$ and relative flow speed $(w)$ during the rotation of the rotor (see Fig. 2). The magnitude of the angles can be largely higher than the stall angle and is governed by the tip-speed ratio $\lambda$. In particular, water turbines that feature a high-solidity design are prone to dynamic stall. Their high solidity will result in a low- $\lambda$ best-efficiency point. The alternating loads with peaks from the profile stall have a significant impact on the lifetime of the turbine.

Studies with scope to optimise the vertical-axis turbines concentrate mostly on the search for an optimal blade geometry [4 10]. Different studies mostly based on computational fluid dynamics (CFD) with fully automatised simulations [6] coupled with genetic algorithms were carried out [7, 8], e.g. Daróczy et al. coupled genetic algorithms with two-dimensional unsteady Reynolds-averaged Navier-Stokes-based CFD simulations. Significant improvements in the performance with an optimised blade shape were reported. Other studies were based on experiments coupled with simulations $[9,10]$. Also, the guiding vanes and collector devices are subject of the ongoing research [11]. 


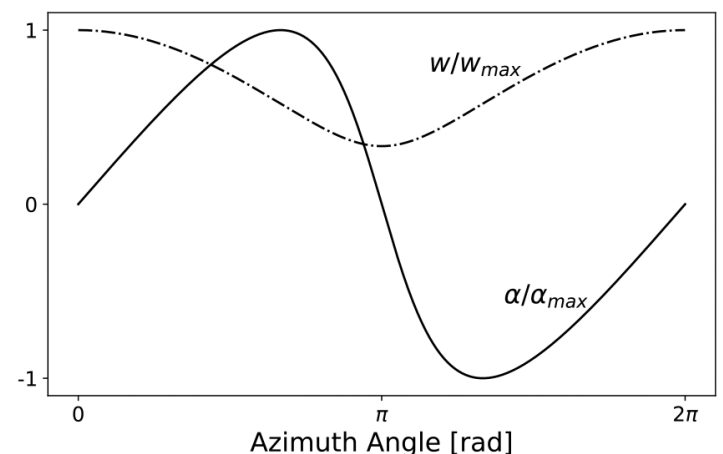

Fig. 2 Normalised angle of incidence $\alpha / \alpha_{\max }$ and relative speed $w / w_{\max }$ at a turbine blade for half rotor turn

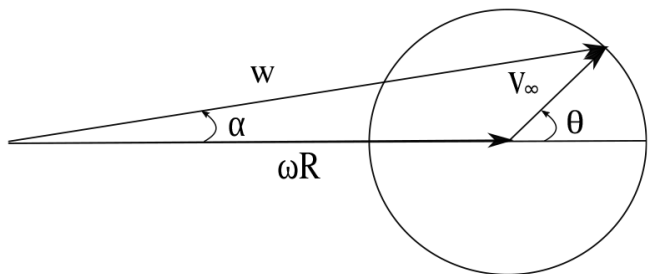

Fig. 3 Speed triangle at a turbine blade, from [30]. The relative velocity $\boldsymbol{w}$ is the vector sum of the inlet velocity $\boldsymbol{v}_{\infty}$ and the tangential velocity $\boldsymbol{\omega} \times \boldsymbol{R}$ resulting from the rotation. The angles in the triangle change with the azimuth rotor angle $\theta$

Besides the optimisation of the blade geometry, other approaches improve the hydrodynamics of the blades by balancing the flow characteristics with blade pitch methods. The aim of the pitch mechanism is to control the angle of incidence $\alpha$ in order to avoid a deep stall regime in the turbine rotor. Pitch control methods for horizontal-axis water turbines (HAWTs) are already well researched, e.g. [12-16]. They depend mainly on free stream $v_{\infty}$ and angular velocity $\omega$, and adjust the angle of incidence $\alpha$ for a full rotation. Pitch control in VAWT is much more challenging, since the pitch angle $\gamma$ depends not only on $v_{\infty}$ and $\omega$ but also on the current azimuth angle $\theta$, because $\alpha$ varies with $\theta$ (see Fig. 2). Consequently, it is required to follow a high dynamic pitch angle trajectory. Unless the structure is adaptive (as studied in $[3,17$, 18]) this pitch mechanism can be controlled either in a passive or active way. It allows one to increase the efficiency and self-starting capabilities of VAWT, e.g. [19-21]. Several studies conclude that active pitch control has a high potential to improve turbine characteristics [20,21]. Most active approaches consist of a central actuator in conjunction with a mechanical transmission system (e.g. a swash plate inspired by helicopter rotors). These mechanisms require a high level of maintenance and are more prone to failure than the turbine itself [22]. A less-explored and recent approach is the use of decentralised actors [23-27]. Kavade investigated the optimal blade pitch trajectory analytically for multiple operating points with the momentum-based model [26]. The obtained optimum pitch trajectory displays similarities to the results obtained in the study at hand, in particular the maximum pitch angle of about $20-25^{\circ}$. However, the orders of magnitude of the turbine size and reduced frequency do not match the present setup, so that a direct comparison may not be meaningful. The obtained power coefficient $c_{p}=0.49$ is very high. Erfort et al. [27] investigated the optimal pitch trajectory with a momentum-based model coupled with the genetic algorithm. The objective was to minimise the torque ripple without performance loss. A 4\% ripple reduction was obtained. The study presented here goes beyond the constraints of that setup, which prescribed a sinusoidal pitch motion.

The present contribution introduces an experimental method to achieve the constraints and objectives for a decentralised active pitch system, using a parametric research approach with a hardware-in-the-loop technique and a strongly simplified model of such a turbine. The method will provide an experimentally based estimation of the optimal pitch angle $\gamma$ for a VAWT in a specific operation point. The benefit of the present contribution is the coupling of a hardware-in-the-loop-based experimental approach with a known surrogate model of a VAWT. This new methodology will allow one to determine the optimal blade pitch with low computational and time costs along with simple hardware resources.

\section{Methodology}

\subsection{Fluid mechanical model}

The relevant fluid mechanical characteristics of a VAWT are detailed in [3]. They are described with dimensionless parameters such as solidity $\sigma$ of the turbine, tip-speed ratio $\lambda$ and reduced frequency $k$.

The tip-speed ratio $\lambda$ governs both the magnitude of the maximum angle of incidence $\alpha_{\max }$ and the variation of $\alpha$ as the turbine rotates (the shape of the $\alpha-\theta$ trajectory, where $\theta$ is the azimuth angle of the turbine). The maximum power point of the most water turbines is found in an interval of $\lambda$ from 2 to 3 , which is significantly lower than for the wind turbines, whose solidity is lower. This leads to $\alpha_{\max }$ ranging from $20^{\circ}$ to $30^{\circ}$ which far exceeds

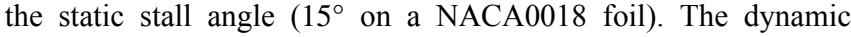
processes on the blade produce a very complex flow, which is from the intensive research interest e.g. [28, 29]. Nevertheless, the value of $\alpha$ without the blade pitch mechanism and for a chord aligned with the circle of rotation follow given functions of $\theta$ and $\lambda$ (1), which can be derived by geometrical relations (see Fig. 3):

$$
\alpha=\arctan \left(\frac{\sin \theta}{\lambda+\cos \theta}\right)
$$

The reduced frequency $k$ is cognate to the Strouhal number - an important dimensionless parameter to quantify unsteady processes in fluid dynamics. The reduced frequency relates the time scale of the variation of the angle of incidence $\left(\alpha_{\max } / \dot{\alpha}\right)$ to the time scale of the vortex convection past the turbine blade $\left(C / 2 v_{\infty}\right)$ with $C$ being the blade chord. It is, in combination with $\alpha_{\max }$, a measure for the flow characteristics and determines the grade of the dynamics and the characteristics of a possible profile stall (see (2) [30]). The left part of (2) expresses $k$ for a straight flow, while the right part is valid for a rotating flow like it appears in a helicopter rotor [30, 31]:

$$
k=\frac{C \dot{\alpha}_{\max }}{2 v_{\infty} \alpha_{\max }}=\frac{C \dot{\alpha}_{\max }}{\omega_{\mathrm{T}} 2 R \alpha_{\max }}
$$

In (3) (reproduced from [30]), $k_{\mathrm{T}}$ is expressed for a VAWT with respect to the non-sinusoidal variation of the angle of incidence at the blade level for a rotating flow. The ratio of blade chord $C$ to turbine radius $R$ is featured, which is equal to the solidity $\sigma$ of a single-bladed turbine, a major parameter for the turbine characteristics along with $\lambda$ which is as well part of the equation. In consequence, the design of a VAWT can be mapped only with two parameters, $\lambda$ and $k$ (see Fig. 4):

$$
k_{\mathrm{T}}=\frac{C}{2 R(\lambda-1) \arctan \left[\left(\lambda^{2}-1\right)^{-(1 / 2)}\right]}
$$

A single-blade turbine can be considered as one oscillating hydrofoil placed in a flow with alternating speed. It performs a rotative motion given from the trajectory in (1) [32] with an average oscillation frequency $f_{\mathrm{o}}$. In that case, the blade-blade interaction in the turbine is neglected. To transpose the reduced frequency definition for a turbine $k_{\mathrm{T}}$, with blades facing an average upwind velocity about $\omega_{\mathrm{T}} R$, to an oscillating foil, facing an upwind velocity equal to the tunnel velocity $v_{\mathrm{ch}}$, the following relations are used (5) [3]:

$$
\omega_{\mathrm{T}} R=v_{\mathrm{ch}} \text { and } f_{\mathrm{o}}=\omega_{\mathrm{T}} / 2 \pi=1 / T_{\mathrm{osc}}
$$




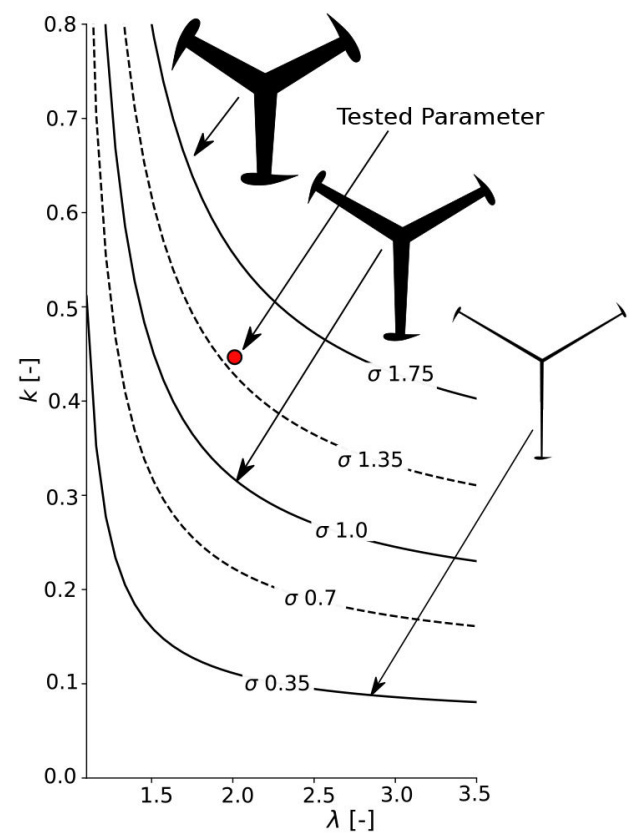

Fig. 4 Influence of tip-speed ratio $\lambda$ and reduced frequency $k$ on the turbine design and the scope of the study. Thin structures are characterised with low $k$, while it raises with $\sigma$, the solidity of the structure. Furthermore, $k$ reduces with increasing $\lambda$ as the maximum angle reduces. The reduced frequency chosen for the present study maps the results to a specific turbine design ([3])

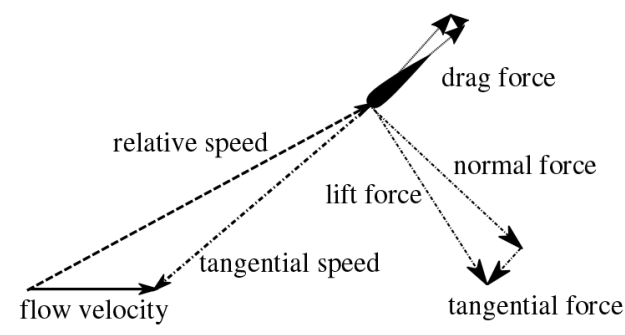

Fig. 5 Forces and speed triangle at a turbine blade. Only the tangential force (sinusoidal share) leads to thrust, the higher cosine share generates structural loads which have to be assimilated by the structure

where $f_{\mathrm{o}}$ is the frequency of the turbine rotation (from the angular velocity $\omega_{\mathrm{T}}$ ) and also the frequency of the blade oscillation in the tunnel given by the oscillation period $T_{\mathrm{osc}}$. For a comparison of the results, the reduced frequency $k$ is redefined for a VAWT trajectory in a non-rotating reference frame $k_{\mathrm{o}}$ by (5) [3]

$$
k_{\mathrm{o}}=\frac{\pi f_{\mathrm{o}} C}{v_{\mathrm{ch}}(\lambda-1) \arctan \left[\left(\lambda^{2}-1\right)^{-(1 / 2)}\right]}
$$

By iteratively adding a pitch angle $\gamma$ to the original trajectory of $\alpha$ and capturing the resulting turbine driving thrust, the best motion law can be determined experimentally. With the present experimental approach, a setup in the hydraulic tunnel of the LEGI Lab in Grenoble (see Section 2.3), the alternating flow speed (see Fig. 2) is not realisable and the possibly related unsteady effects are not reproduced. Nevertheless, it is probable that the flow unsteadiness due to the variation of the incidence alpha is predominant compared to the one due to the variation of the upstream velocity. However, this difference has to be considered in the interpretation of the results. The hydrodynamic forces are normalised with the corresponding instantaneous inlet flow speed $v_{\text {ch }}$ to lift coefficient $c_{\mathrm{L}}$ and drag coefficient $c_{\mathrm{D}}$ by (6) and (7):

$$
\begin{aligned}
c_{\mathrm{L}} & =\frac{F_{y}}{(1 / 2) S \rho v_{\mathrm{ch}}^{2}} \\
c_{\mathrm{D}} & =\frac{F_{x}}{(1 / 2) S \rho v_{\mathrm{ch}}^{2}}
\end{aligned}
$$

where $F_{x y}$ are the forces in the corresponding axis, $\rho$ is the density of the water and $S$ represents the blade surface. The projection of lift and drag on the rotation tangent contribute to the driving thrust, so that the turbine's driving thrust coefficient $c_{\mathrm{T}}$ may be expressed as (8):

$$
c_{\mathrm{T}}=c_{\mathrm{L}} \sin \alpha-c_{\mathrm{D}} \cos \alpha
$$

$c_{\mathrm{T}}$ can be used to compare different motion laws for an active pitch mechanism. The driving thrust coefficient is directly related to the power of the turbine and is low compared to the normal force coefficient (Fig. 5), while the normal forces only generate structural loads on the device. A more detailed description of the hydrodynamic model can be found in [3].

\subsection{Optimisation method}

No reliable analytical model or mathematical description is available which would allow one to define an optimal pitch trajectory for a given turbine. The existing code like momentumbased methods requires experimentally acquired force coefficients and shows insufficient results for the dynamic characteristics and (dynamic) stall. In consequence, the interaction between the variations in the pitch trajectory $\gamma$ and the corresponding forces were evaluated experimentally with a design-of-experiment methodology for a turbine characterised by the reduced frequency $k$ of the oscillation and tip-speed ratio $\lambda$. In order to implement the experimental model, the blade was set in forced oscillation with a servo drive following the trajectory $\alpha$ (1). To emulate the pitch motion, an additional pitch angle $\gamma$ was added to the original trajectory of $\alpha$ (see Fig. 6). The optimal function of $\gamma$ balances the blade motion in such a way that the mean thrust $C_{\mathrm{T}}(8)$ is maximised. A new trajectory $\alpha^{\prime}=\alpha+\gamma$ defines this optimised motion law for the blade oscillation with the pitch control.

In the experiment, the analytical description of the pitched trajectory is replaced by a spline defined through a set of parameters consisting of five knots. In the present setup, two knots (start and endpoint) have fixed values. The variation of the three remaining parameters $\left(x_{1}, x_{2}, x_{3}\right)$ allows one to define all realisable trajectories for the setup, as shown in Fig. 7. The aim of the optimisation is to maximise the objective function defined by (9). The knots are fully dependent on each other - the dynamics of the flow and occurrence of separation depend on the variation in time of the angle of incidence and pitch motion. In consequence, no 


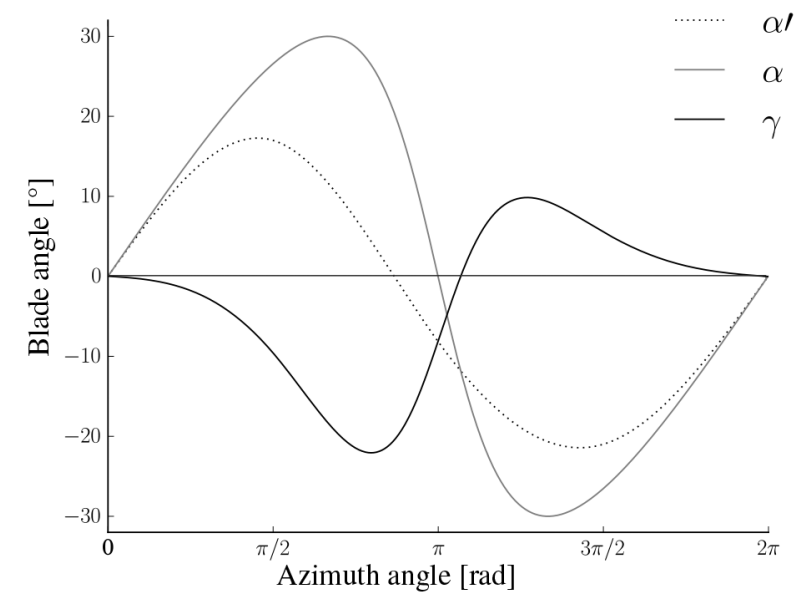

Fig. 6 Presumptive diagram of the probable relation between original angle of incidence $\alpha$, pitch angle $\gamma$ and resulting angle of incidence $\alpha^{\prime}$

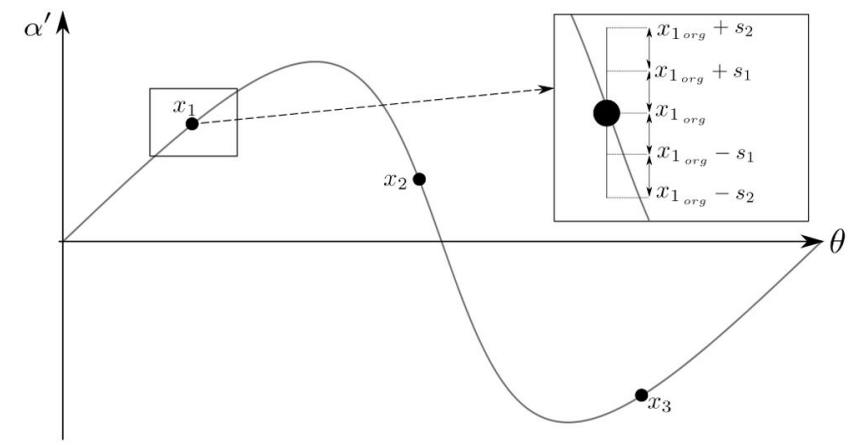

Fig. 7 Generation of the knots for the base spline. The start and end points of the spline are always fixed to zero, while the three parameters $x_{1}, x_{2}, x_{3}$ are varied in the inner steps $S_{i}$ in the positive and negative directions

gradient-based method or successive optimisation of each parameter is suitable for the optimisation task. Instead, a factorial design of the experiment or the so-called fully crossed design was selected.

The inspection of one set of parameters required only a short amount of time ( $\sim 35 \mathrm{~s})$, this is why the more sophisticated methods such as a genetic algorithm was not implemented in this state. In further studies, more elaborated tools such as the laboratory's inhouse optimiser OPAL++ [7] will enable the examination of a whole range of possible turbine designs in a shorter time. The objective function OF (9) is defined by the driving thrust of the turbine (see Section 2.1) which is expressed as a function of the thrust coefficient $c_{\mathrm{T}}$. The integral for one oscillation period has to be maximised:

$$
\mathrm{OF}=\int_{0}^{2 \pi}\left(c_{\mathrm{L}} \sin \alpha+c_{\mathrm{D}} \cos \alpha\right) \mathrm{d} \theta
$$

In order to provide simple and robust setup, the optimisation is carried out in a scaled approach, with a step-wise reduction of the parameter bandwidth. At the beginning of each outer step, a start curve is defined. The three corresponding knots $\left(x_{n_{\text {org }}}\right)$ of the function are varied with inner steps $s_{i}$ (see Fig. 7), so that the $x_{n_{m}}$ values are generated as follows:

$$
x_{n_{m}}=x_{n_{\mathrm{org}}} \pm s_{i} \text { stepsize }
$$

This leads to a matrix of $n$ parameters and $m$ inner steps. In the existing setup with $n=4$ and $m=3$, the size is $4^{3}=64$ values for each outer step. The practical implementation consists of a set of Python scripts, which allows one to fully automate the each outer step. For each inner step, a spline was generated, normalised, processed and transmitted to the drive controller by a custom communication library. The drive system oscillated the blade

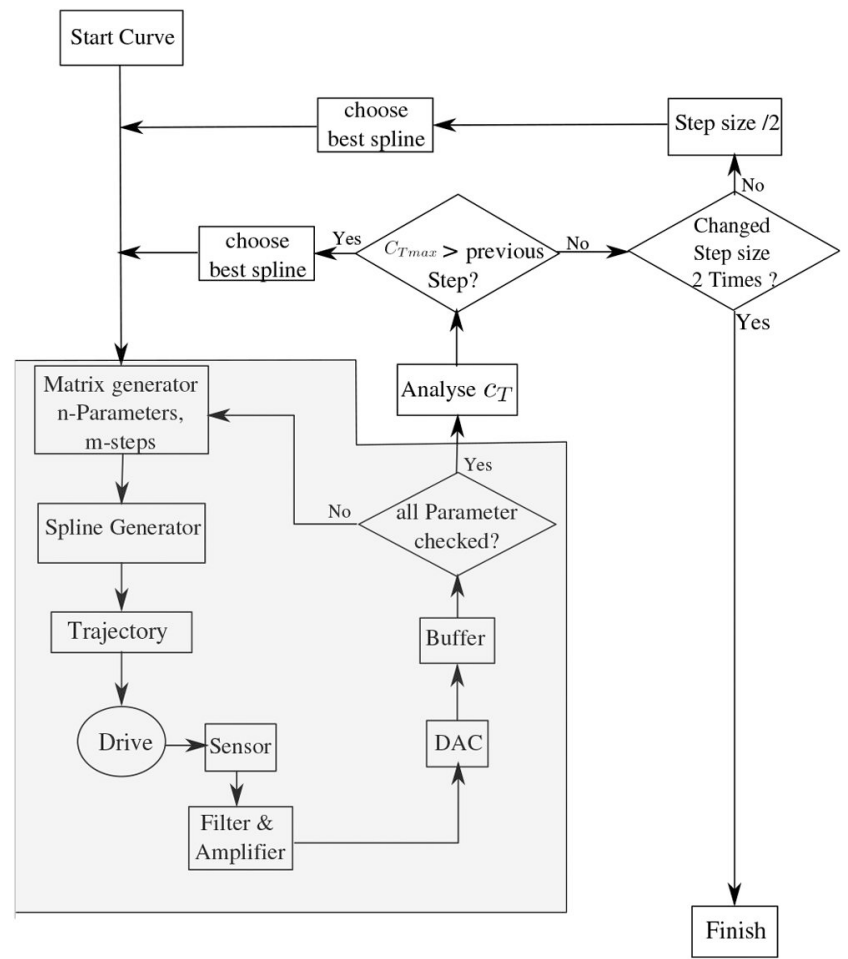

Fig. 8 Flowchart of the hardware loop-based optimisation with inner (dark grey) and outer steps (light grey). The step size is changed if the objective function did not improve in the last step, after two successive steps without improvement the optimisation is stopped

corresponding to the current spline for $t=2500 \mathrm{~ms}$. After an idle time of $1 \mathrm{~s}$ to establish the flow condition, 1000 scans of each input were streamed with a scan rate of $1 \mathrm{kHz}$ from the buffer of the data acquisition card (DAC) and saved as a.json file dump. Once all combinations were completed, the data was processed to detect the best spline of the set. This spline fed the next outer step of the optimisation process with successively small step sizes, until the results converged (see Fig. 8).

\subsection{Experimental setup}

The experimental setup is shown in Fig. 9 [3]. The experiment took place in the closed-loop hydraulic channel in the LEGI Laboratories, Grenoble (see [33] for a detailed description). The control section's dimensions were $1000 \times 280 \times 175 \mathrm{~mm}^{3}$ in the $X$ $Y-Z$ coordinates of Fig. 9. The foil was milled from aluminium into a NACA0018 profile, with rotation centre positioned at the quarter chord. The chord-based Reynolds number for the setup with $3.5 \mathrm{~m} / \mathrm{s}$ inlet velocity and a chord length of $0.066 \mathrm{~m}$ is $\mathrm{Re} \simeq 2 \times 10^{5}$.

The electrical drive system consisted of a $5 \mathrm{~kW}$ brushless servo machine equipped with a resolver for position feedback and drive inverter. The drive inertia was $0.012 \mathrm{~kg} \mathrm{~m}^{2}$ and the peak torque was $143 \mathrm{~N} \mathrm{~m}$. A submersible six-axis load cell (SRI-M3714BP) was mounted between the shaft stub from the transmission side and the profile to measure forces and torques applied on the blade during the experiment. The cell was able to measure forces reaching 800 $\mathrm{N}$ in the $X$ and $Y$ directions, and $1600 \mathrm{~N}$ in the $Z$ direction. The range of torque measurement in all axes is $44 \mathrm{~N} \mathrm{~m}$. The sensor was conceptionally oversized due to the cyclic loads to prevent fatigue failure. A DAC gathered the position feedback as quadrature encoder signal, as well as the flow speed, water temperature, forces and torques from the six-axis load cell as analogue signals. A custom-built instrument signal amplifier was designed with six analogue inputs from the load cells.

The standalone DAC (Labjack T7-pro), with a maximum overall sample rate of $100 \mathrm{kHz}$, acquired six analogue inputs from the load cell, three digital inputs from the encoder (16-bit resolution for each channel) and three signals from the water tunnel. The DAC communicated and streamed the data to the 

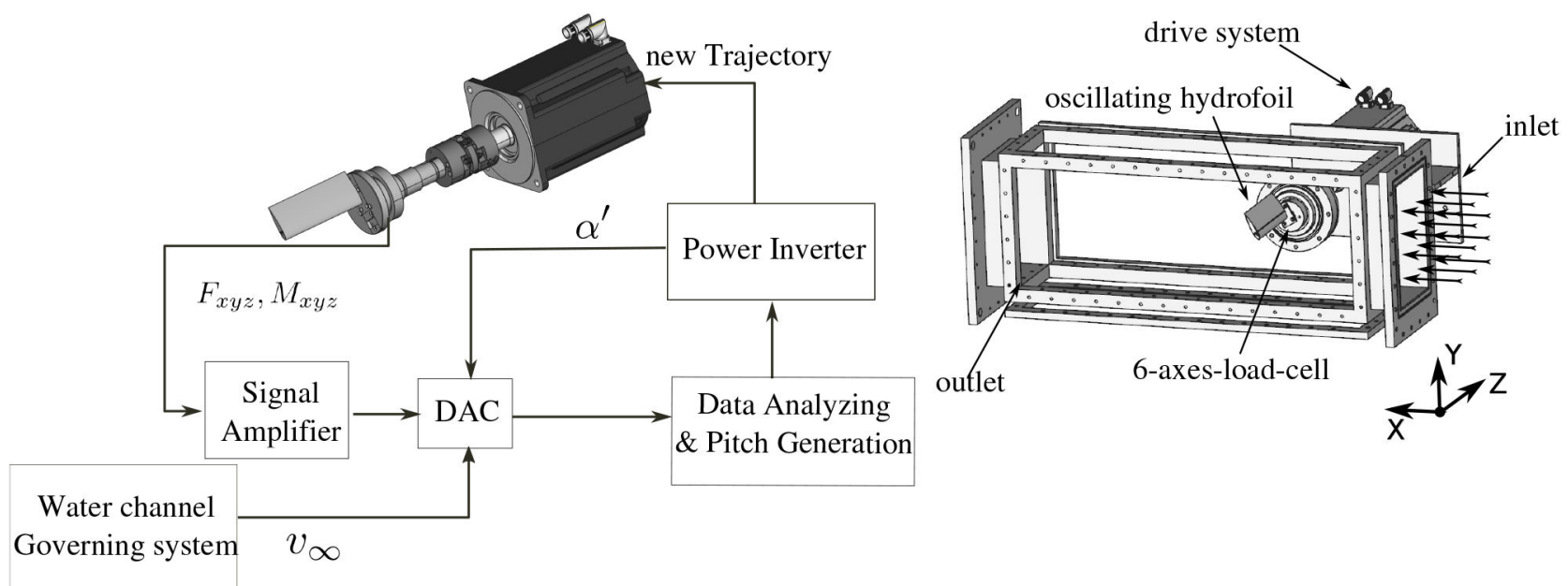

Fig. 9 Experimental setup at the LEGI closed-loop water tunnel. The hydrofoil performs non-harmonic forced oscillations induced from the drive systems with parallel force and torque measurements from the fully submerged load cell

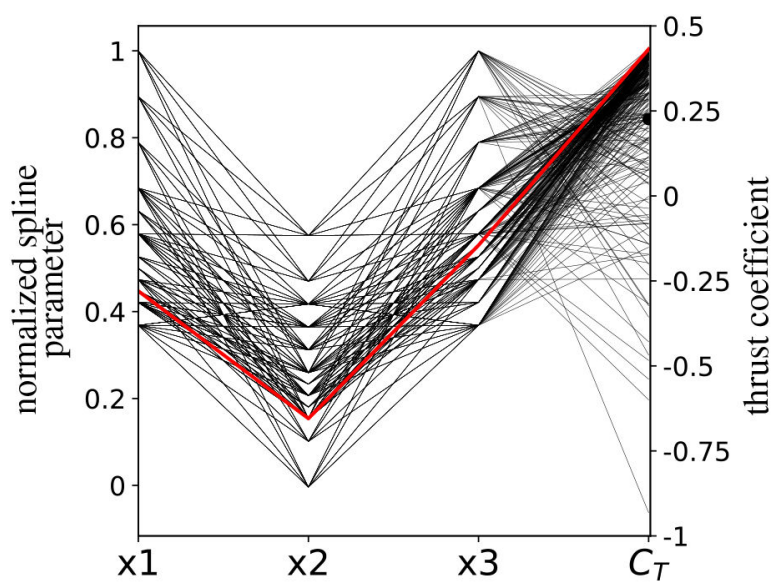

Fig. 10 Parameter variation and corresponding thrust coefficient for the optimisation

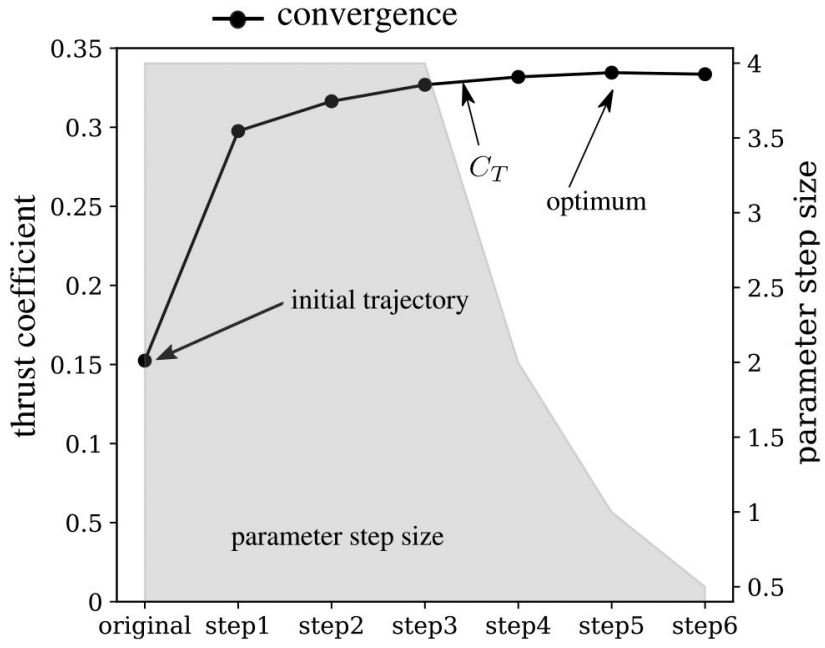

Fig. 11 Convergence of the multi-scale optimisation. The scaling parameter step (in ${ }^{\circ} \mathrm{deg}$ ) is displayed in light grey in the background

controller PC over TCP/IP protocol (Ethernet) to achieve sufficient streaming rates. The data stream was processed in the controller PC using Python scripts. Also, the power inverter was governed over Telnet protocol and Ethernet connection using a custom Python library. The library was published under GPLv3 [34] over a bitbucket repository. The details of the experiment's workflow and the algorithm topology are shown in Figs. 8 and 9. The software allows one to submit arbitrary periodic rotational motions in its mathematical description. The resulting trajectory is converted to a normalised curve and saved as a CSV file, which is finally transmitted to the drive. Care has to be taken in the generation of the reference trajectory for minimising the derivative of acceleration (jerk). An acceleration discontinuity has to be avoided in the transition between two periods.

Regarding the measurement uncertainty, the drive system is equipped with a resolver with $0.0439^{\circ}$ angular resolution. Adding the uncertainty for the zero angle calibration of $0.014^{\circ}$ given by a level with accuracy $0.25 \mathrm{~mm} / \mathrm{m}$, the overall set point accuracy is given by $0.058^{\circ}$.

The load cell chosen was with respect to the cyclic loads. A safety factor of three was used with respect to the expected peak forces and moments. The force measurement system uncertainty was measured with standard weights. It is negligible for a static load on a single axis, in the range of the experiment with $-250 \mathrm{~N}<F_{y}<250 \mathrm{~N}$ and $0 \mathrm{~N}<F_{x}<150 \mathrm{~N}$. The maximum error is $<0.009 \%$ relative to full scale $(800 \mathrm{~N})$ and, respectively, of $0.05-4.8 \%$ relative error to a load of 2 to $0.1 \mathrm{~kg}$ standard weights.

The significant parameter is the signal crosstalk, which is specified by the manufacturer to a full scale. However, he stated that the uncertainty could also be calculated based on the reading. The uncertainty ranges from $0.3 \%$ of $X$ reading for the $Y$-axis, maximum loaded with $800 \mathrm{~N}$, up to $1.82 \%$ of $X$ reading for the $Z$ axis, maximum loaded with $1600 \mathrm{~N}$. The dynamic characteristics of the sensor were given with $0.26 \%$ hysteresis on reading, with maximum load of $800 \mathrm{~N}$ for $X$ and $Y$.

The present study focuses on one working point for one turbine design: the reduced frequency was kept constant at $k=0.45$ as well as the tip-speed ratio at $\lambda=2(2)$. This design point is typical for a low- $\lambda$, high- $\sigma$ water turbine. The experimental set corresponds to $\alpha_{\max }=30^{\circ}$ and an oscillation frequency $f \simeq 3.5 \mathrm{~Hz}$ (see Fig. 4). Variations of $f$ (with dependence on $\alpha_{\max }$ ) were observed and considered to be negligible.

\section{Results and discussion}

The parameter variation and corresponding output are shown in Fig. 10. The parameters were normalised for better visualisation. $x_{1}$ $\left(14-38^{\circ}\right)$ is divided by the maximum angle $\alpha_{\max }, x_{3}\left(-38^{\circ}\right.$ to $\left.-14^{\circ}\right)$ by the minimum $\alpha_{\min }$, while $x_{2}\left(-14^{\circ}\right.$ to $\left.8^{\circ}\right)$ is first shifted to positive values and then divided by the maximum of $x_{2}$. The diagram shows how the method moves the parameters towards the optimal spline knots with high $c_{\mathrm{T}}$ (higher parameter concentration near to the optimum). A large spread with very disparate values is only observed for few parameter sets (in the first outer step), while the later outer steps result in a much narrower range.

Fig. 11 shows the maximum thrust coefficient for each outer step of the optimisation. The values behave asymptotically to an optimum of $c_{\mathrm{T}} \simeq 0.333$, an improvement of $122 \%$ over the value found on the initial trajectory without an active pitch. The optimisation converged after five outer steps (corresponding to 320 


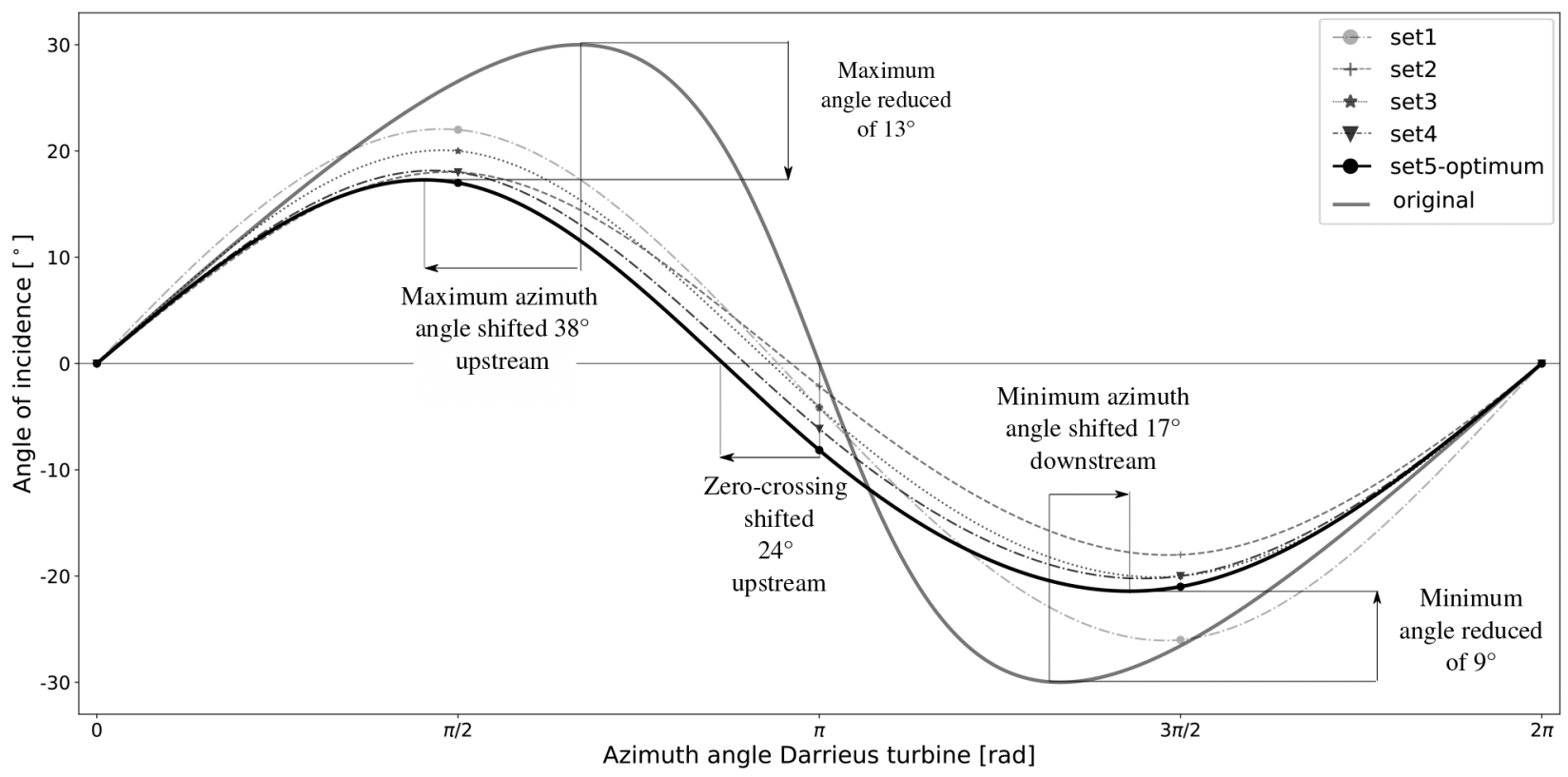

Fig. 12 Original trajectory and the evolution of the motion law till the optimum. The motion law becomes smoother with smaller angle change rates $\omega$. Also, the transition point from positive to negative $\alpha^{\prime}$ is shifted

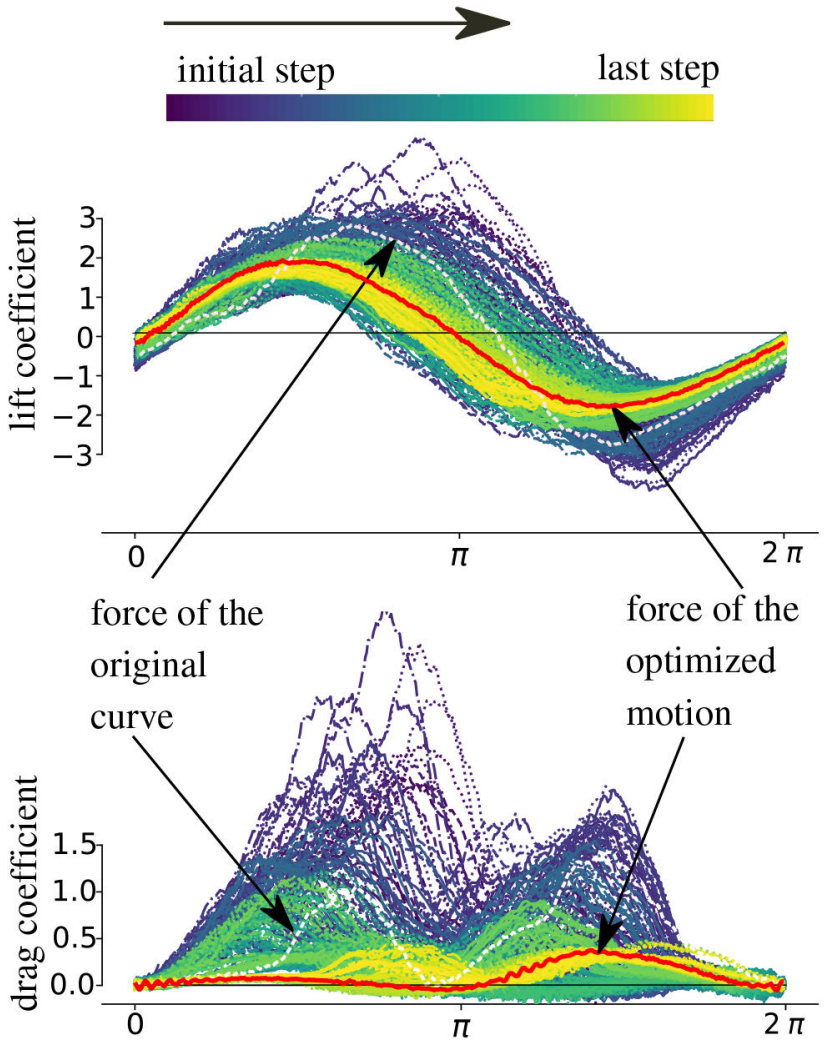

Fig. 13 Lift and drag forces for the parameter variation. The original curve is highly unsteady with overshooting drag. The parameter variation is mapped starting from violet curves and is shifted to brighter colours for each outer step. The variations are successively smoothed out till the optimum is reached, which show very low drag and an almost symmetric lift trajectory

variants). The inner step size is scaled down from $4^{\circ}$ (steps $\left.1-3\right)$ to $1^{\circ}$ (step 5). No improvement of $c_{\mathrm{T}}$ was found at the sixth outer step, which was refined to a step size of $0.5^{\circ}$. The final result was obtained after $5 \mathrm{~h}$, of which $3 \mathrm{~h} 15$ min was pure run time.

Fig. 12 shows the evolution of the motion law during the optimisation process. In the original curve, a slow-motion phase around $\theta=0$ is followed by a high-speed phase between $\pi / 2$ and $3 \pi / 2$ radians. $\alpha_{\max }$ is $30^{\circ}$, a deep dynamic stall is expected for a significant bandwidth of $k$, with partially improved lift but high drag forces.

In the obtained optimal motion law, the magnitude of the maximum and minimum angles was significantly reduced (a $13^{\circ}$ reduction in the ascending and $9^{\circ}$ in the descending branches). The high-speed phase was eased, with the maxima brought forward and minima shifted to a later point, so that the time interval separating the extrema was increased. The zero-crossing point (where $\alpha=0^{\circ}$ ) was also brought forward in time; this may be partly compensating for the hysteresis of the reattachment of the flow and may reduce inertial effects within the flow.

Fig. 13 displays the lift and drag forces on the blade for the whole parameter set. As expected from the motion law, the optimisation algorithm shifted the transition point of the force (lift switches from positive to negative) to an earlier azimuth angle. The bandwidth of $c_{\mathrm{L}}$ was very large (maximum from 1.5 to 5), with the oscillating motion permitting much larger values than for static cases. The deep dynamic stall was observed for the trajectories with high lift coefficients, as indicated by the abrupt rise of the corresponding drag. These phenomena were not appearing for trajectories with lower lift (and so lower maximum angles of incidence). It can be seen that the optimised solution displays sharply reduced values for drag across the entire range of azimuth angles, at the cost of sensibly reduced values of lift compared to the starting point.

\section{Conclusion}

The enormous potential of active pitch mechanisms for increasing the power output of VAWT became visible in the current study. The objective for a VAWT pitch control is to find an optimum between low drag (no deep dynamic stall) and high lift (either close to the onset of or with light dynamic stall), as applied on the blades throughout their path. However, the results of the study showed that the losses incurred through drag due to profile dynamic stall had a substantially higher impact than the corresponding extreme values in the lift.

The method introduced in this study showed promising results and was well suited to study the operating bandwidth of independently mounted actuators for the optimum pitch control. The benefit of the presented methodology is the possibility to study a complete range of turbine designs by the variation of two parameters $(\lambda$ and $k$ ), with a very short runtime compared to numerical models [3]. An optimal pitch trajectory delivering a significant improvement in the thrust coefficient was obtained in a few hours only. The coupling of a hardware-in-the-loop-based, 
full-factorial optimisation with a surrogate model of a VAWT minimises the investigation time and costs, compared to the common methodologies, such as CFD or experiments on a complete VAWT turbine. It allows to qualitatively research the optimum blade pitch.

Further work will include the comparison with numerical models and experimental assessment of its transferability. In particular, the scope of the study will be expanded to include a broader range of turbine designs and the visualisation of the corresponding flow fields.

Based on the measured forces, torques and moments, it will be possible to size and build the necessary actuators to implement a blade pitch control of the HAWT at the next steps. The necessary working range for a pitch mechanism is $22^{\circ}$ (according to Fig. 12). In consequence, a deployed actuator could be either a limited angle torque motor [35] or a linear motor, e.g. a tubular-type drive, which both would have the potential to be integrated into the turbine structure.

\section{Acknowledgments}

The authors are grateful to the Laboratoire des Écoulements Géophysiques et Industriels (LEGI) for the opportunity to use the water channel and the laboratories in Grenoble. Furthermore, the authors thank Michel Riondet, Jean-Bastien Carat, Sebastian Bode, Thomas Schallschmidt, Olivier Cleynen and the workshops in Grenoble and Magdeburg for their support and contributions to the experiment.

\section{References}

[1] Whittlesey, R.W., Liska, S., Dabiri, J.O.: 'Fish schooling as a basis for vertical axis wind turbine farm design', Bioinspiration Biomimetics, 2010, 5, (3), p. 035005

[2] Dabiri, J.O.: 'Potential order-of-magnitude enhancement of wind farm power density via counter- rotating vertical-axis wind turbine arrays', J. Renew. Sustain. Energy, 2011, 3, p. 043104

[3] Hoerner, S., Abbaszadeh, S., Maître, T., et al.: 'Experimental evaluation of the characteristics of highly flexible structures in vertical-axis water turbines', J. Fluid Struct., 2019, 88, pp. 13-30

[4] Hashem, I., Mohamed, M.H., Hafiz, A.A.: 'Unsteady investigation of smallscale shrouded wind turbine with a brimmed diffuser', Energy, 2016, 136, (118), pp. 345-368

[5] Bedon, G., Bett, S.D., Benini, E.: 'Performance-optimized airfoil for Darrieus wind turbines', Renew. Energy, 2016, 94, pp. 328-340

[6] Carrigan, T.J., Dennis, B.H., Han, Z.X., et al.: 'Aerodynamic shape optimization of a vertical-axis wind turbine using differential evolution', ISRN Renew. Energy, 2012, 2012, pp. 1-16

[7] Daróczy, L., Janiga, G., Thévenin, D.: 'Computational fluid dynamics based shape optimization of airfoil geometry for an H-rotor using a genetic algorithm', Eng. Optim., 2018, 50, (9), pp. 1483-1499

[8] Ivanov, T.D., Simonović, A.M., Svorcan, J.S., et al.: 'VAWT optimization using genetic algorithm and CST airfoil parameterization', FME Trans., 2017, 45, pp. 26-31

[9] Ragni, D., Ferreira, C.F., Barone, M.: 'Experimental and numerical investigation of an optimized airfoil for vertical axis wind turbines'. 32nd ASME Wind Energy Symp. AIAA SciTech (American Institute of Aeronautics and Astronautics), National Harbor, Maryland, 2014, pp. 1-10

[10] Yamazaki, W.: 'Experiment / simulation integrated shape optimization using variable fidelity Kriging model approach', Adv. Mech, Des. Syst. Manuf., 2017, 11, (5), pp. 1-9

[11] Hashem, I., Mohamed, M.H.: 'Aerodynamic performance enhancements of H-rotor Darrieus wind turbine', Energy, 2018, 142, pp. 531-545
[12] Munteanu, I., Bratcu, A.I., Bacha, S., et al.: 'Hardware-in-the-loop-based simulator for a class of variable-speed wind energy conversion systems: design and performance assessment', IEEE Trans. Energy Convers., 2010, 25, (2), pp. 564-576

[13] Qiao, Y., Han, S., Deng, Y., et al.: 'Research on variable pitch control strategy of wind turbine for tower vibration reduction', J. Eng., 2017, 2017, (13), pp. 2005-2008

[14] Soliman, M., Malik, O.P., Westwick, D.T.: 'Multiple model multiple-input multiple-output predictive control for variable speed variable pitch wind energy conversion systems', IET Renew. Power Gener., 2011, 5, (2), pp. 124 136

[15] Liu, H., Wang, Y., Tang, Q., et al.: 'Individual pitch control strategy of wind turbine to reduce load fluctuations and torque ripples'. Int. Conf. on Renewable Power Generation, Beijing, October 2015

[16] Han, B., Zhou, L., Yang, F., et al.: 'Individual pitch controller based on fuzzy logic control for wind turbine load mitigation', IET Renew. Power Gener. 2016, 10, (5), pp. 687-693

[17] Herman, D., Gundersen, Z.: 'A novel flexible foil vertical axis turbine for river, ocean and tidal applications', Appl. Energy, 2015, 151, pp. 60-66

[18] MacPhee, D.W., Beyene, A.: 'Fluid-structure interaction analysis of morphing vertical axis wind turbine', J. Fluid Struct., 2016, 60, pp. 143-159

[19] Mauri, M., Bayati, I., Belloli, M.: 'Design and realisation of a highperformance active pitch-controlled H-darrieus VAWT for urban installations'. 3rd Renewable Power Generation Conf., Naples, Italy, 2014

[20] Lazauskas, L., Kirke, B.K.: 'Modelling passive variable pitch cross flow hydrokinetic turbines to maximize performance and smooth operation', Renew. Energy, 2012, 45, pp. 41-50

[21] Khalid, S.S., Liang, Z., Qi-hu, S., et al.: 'Difference between fixed and variable pitch vertical axis tidal turbine-using CFD analysis in CFX', Res. J. Appl. Sci. Eng. Technol., 2013, 5, (1), pp. 319-325

[22] Ferreira, C.S., Madsen, H.A., Barone, M., et al.: 'Comparison of aerodynamic models for vertical axis wind turbines', J. Phys., Conf. Ser., 2014, 524, p. 012125

[23] Zhang, L., Liang, Y., Liu, X., et al.: 'Effect of blade pitch angle on aerodynamic performance of straight-bladed vertical axis wind turbine', $J$ Central South Univ., 2014, 21, pp. 1417-1427

[24] Paillard, B., Astolfi, J.A., Hauville, F.: 'URANSE simulation of an active variable-pitch cross-flow Darrieus tidal turbine: sinusoidal pitch function investigation', Int. J. Mar. Energy, 2015, 11, pp. 9-26

[25] Liang, Y.-B., Zhang, L.-X., Li, E.-X., et al.: 'Blade pitch control of straightbladed vertical axis wind turbine', J. Central South Univ., 2016, 23, (5), pp. $1106-1114$

[26] Kavade, R.K., Ghanegaonkar, P.M.: 'Effect of best position blade pitching on power coefficient of VAWT at different tip speed ratio by SST \& DMST model', FME Tran., 2018, 46, (4), pp. 560-566

[27] Erfort, G., von Backström, T.W., Venter, G.: 'Reduction in the torque ripple of a vertical axis wind turbine through foil pitching optimization', Wind Eng., 2019, 1, p. 0309524X1983671, DOI: 10.1177/0309524X19836711

[28] Thönnißen, F., Marnett, M., Roidl, B., et al.: 'A numerical analysis to evaluate Betz's law for vertical axis wind turbines', J. Phys., Conf., 2016, 753 , p. 022056

[29] Maître, T., Amet, E.: 'Modelling of the flow in a Darrieus water turbine: wall grid refinement analysis and comparison with experiments', Renew. Energy, 2013, 51, pp. 497-512

[30] Laneville, A., Vittecoq, P.: 'Dynamic stall: the case of the vertical axis wind turbine', J. Sol. Energy Eng. Trans. ASME, 1986, 108, pp. 140-145

[31] McCroskey, W.J.: 'Special opportunities in helicopter aerodynamics' (Springer-Verlag, New York, 1986)

[32] Ly, K.H., Chasteau, V.A.L.: 'Experiments on an oscillating aerofoil and applications to wind-energy converters', J. Energy, 1981, 5, (2), pp. 116-121

[33] LEGI publication team: 'Legi - the hydrodynamic tunnel'. Laboratory webpage, 2018. Available at http://www.legi.grenoble-inp.fr/web/spip.php? article1264\&lang=en

[34] Hoerner, S., Abbaszadeh, S.: 'Kollmorgen akd drive communication library for python'. Software repository, 2018. Available at https://bitbucket.org/ sthoerner/kollmorgen_drive_comm/

[35] Nasiri-Zarandi, R., Mirsalim, M., Cavagnino, A.: 'Analysis, optimization, and prototyping of a brushless DC limited-angle torque-motor with segmented rotor pole tip structure', IEEE Trans. Ind. Electron., 2015, 62, (8), pp. 49854993 